\title{
Comparative thermodynamic studies on substrate and product binding of O-Acetylserine Sulfhydrylase reveals two different ligand recognition modes ${ }^{\dagger}$
}

Shrijita Banerjee, Mary K Ekka and Sangaralingam Kumaran*

\begin{abstract}
Background: The importance of understanding the detailed mechanism of cysteine biosynthesis in bacteria is underscored by the fact that cysteine is the only sulfur donor for all cellular components containing reduced sulfur. O-acetylserine sulfhydrylase (OASS) catalyzes this crucial last step in the cysteine biosynthesis and has been recognized as an important gene for the survival and virulence of pathogenic bacteria. Structural and kinetic studies have contributed to the understanding of mechanistic aspects of OASS, but details of ligand recognition features of OASS are not available. In the absence of any detailed study on the energetics of ligand binding, we have studied the thermodynamics of OASS from Salmonella typhimurium (StOASS), Haemophilus influenzae (HiOASS), and Mycobacterium tuberculosis (MtOASS) binding to their substrate O-acetylserine (OAS), substrate analogue (methionine), and product (cysteine).

Results: Ligand binding properties of three OASS enzymes are studied under defined solution conditions. Both substrate and product binding is an exothermic reaction, but their thermodynamic signatures are very different. Cysteine binding to OASS shows that both enthalpy and entropy contribute significantly to the binding free energy at all temperatures $\left(10-30^{\circ} \mathrm{C}\right)$ examined. The analyses of interaction between OASS with OAS (substrate) or methionine (substrate analogue) revealed a completely different mode of binding. Binding of both OAS and methionine to OASS is dominated by a favorable entropy change, with minor contribution from enthalpy change $\left(\Delta \mathrm{H}_{\mathrm{St}-\mathrm{Met}}=-1.5 \pm 0.1 \mathrm{~kJ} / \mathrm{mol} ; \mathrm{T} \Delta \mathrm{S}_{\mathrm{St}-\mathrm{Met}}=8.2 \mathrm{~kJ} / \mathrm{mol}\right)$ at $20^{\circ} \mathrm{C}$. Our salt dependent ligand binding studies indicate that methionine binding affinity is more sensitive to $[\mathrm{NaCl}]$ as compared to cysteine affinity.

Conclusions: We show that OASS from three different pathogenic bacteria bind substrate and product through two different mechanisms. Results indicate that predominantly entropy driven methionine binding is not mediated through classical hydrophobic binding, instead, may involve desolvation of the polar active site. We speculate that OASS in general, may exhibit two different binding mechanisms for recognizing substrates and products.
\end{abstract}

Keywords: Ligand Binding, Enthalpy, Entropy, Fluorescence, Isothermal Titration Calorimetry

\section{Background}

Cysteine biosynthesis in bacteria is a highly regulated process. Enzymes involved in cysteine biosynthesis function as molecular sensors [1-3]. The de novo biosynthesis of cysteine is catalyzed in two steps. In the first step, serine acetyltransferase (SAT) catalyzes the

\footnotetext{
* Correspondence: skumaran@imtech.res.in

Council of Scientific and Industrial Research, Institute of Microbial Technology, Sector 39-A, Chandigarh, 160036, India
}

formation of OAS from acetylCoA and L-serine. In the second step, OAS is converted to cysteine by O-acetylserine sulfhydrylase (OASS) through elimination of acetate and addition of bisulfide [4-6]. Since cysteine is the only sulfur donor for all cellular components containing reduced sulfur, balanced activity of OASS is very important for growth and survival of the bacterium [7]. In addition to its role in protein structure, cysteine is the precursor for the biosynthesis of a variety of primary
C Biomed Central 
and secondary metabolites like glutathione, vitamin cofactors, anti-oxidants, etc $[8,9]$. The conservation of cysteine biosynthesis genes including OASS in many intracellular pathogens and up regulation of OASS during bacterial infection suggests that OASS is essential for the survival of these pathogens $[10,11]$. Loss of OASS has been shown to affect both the survival fitness of Salmonella under hostile conditions and virulence of the bacterium [12]. Thus, inhibition of OASS using specific inhibitors should result in reduced survival of bacteria inside the macrophage. Absence of OASS homologues in humans makes it an attractive antibacterial drug target. A recent study has exploited the protein-protein interaction properties of OASS and SAT to design small peptides that selectively bind to OASS with higher affinity and inhibit its activity [13].

Crystal structures of OASS from S. typhimurium in the presence and absence of ligands have been determined [14,15]. Three-dimensional structure of OASS from $H$. influenzae has been determined in complex with C-terminal peptide of serine acetyltransferase [16]. Structural studies show that OASS is a homo-dimer and it belongs to the $\beta$-family of PLP-dependent enzymes with one PLP/subunit buried within the protein. In the absence of bound ligand, the coenzyme pyridoxal 5'phosphate (PLP) is bound via a Schiff base to the side chain of Lys41 [14]. Activity of OASS is regulated by its metabolites and other enzymes involved in cysteine metabolism. Spectroscopic properties of active site bound PLP has been exploited to investigate the ligand recognition principles of OASS [17]. Structural and biochemical studies have shown that OASS can bind both cysteine, its product, and methionine, substrate analogue of OAS [15,17-19]. Comparison of crystal structures of methionine bound S. typhimurium OASS (StOASS-met) and cysteine bound E. histolytica OASS (EhOASS-cys) indicates that both amino acids are found in the active site center with their $\alpha$-amino group oriented towards C4A atom of PLP $[15,18,19]$. Therefore, it is expected that both cysteine and methionine may bind to the active site through similar mechanisms. Ligand binding to $S$. typhimurium (StOASS) active site causes conformational changes in the protein [15]. Although structures of complexes offer glimpses of ligand occupied active site of OASS, thermodynamic properties of proteinligand interactions have not been studied. Catalytic mechanism of OASS has been studied in detail [20,21], and studies also show that OASS exhibits both substrate and product inhibition [6]. Further, biochemical properties of cysteine synthesis enzymes are influenced by binding of substrates, products, small ions, and other proteins $[3,15,17,18]$. Understanding the energetics of interactions of these small molecules with proteins would provide information on regulatory features such as binding modes for ligands. Quantitative characterization of energetics of protein-ligand interactions would provide additional information which is necessary to understand the details of ligand recognition features of an enzyme.

Studies on biochemical characterization of OASS have used mostly steady state as well as pre-steady state kinetic approaches to understand the catalytic mechanism. Physiological mechanism of an enzyme deduced only from kinetic studies is incomplete in the absence of information on ligand binding properties. Although structural studies have provided information about residues that mediate ligand binding, ligand recognition mechanisms are studied using thermodynamic and kinetic approaches [22]. Isothermal titration calorimetry is the most direct method for the determination of heats of binding $\left(\Delta \mathrm{H}_{\text {bind }}\right)$ and binding free energy $\left(\Delta \mathrm{G}_{\text {bind }}\right)$ upon protein ligand binding [23]. In the absence of any detailed study to understand the energetics of substrate and product interaction with OASS, we present here a detailed thermodynamic description of ligand binding by OASS from S. typhimurium, M. tuberculosis, and $H$. influenzae. We focused on studying the interaction of OASS with cysteine, O-acetyl serine (OAS), and methionine as a function of temperature and determined the relative contributions of enthalpy $(\Delta H)$ and entropy $(\Delta S)$ to the binding free energy. We observed a predominantly entropy driven binding for substrate and substrate analogue. We also examined these protein-ligand interactions as a function of salt concentrations and studied the salt dependency of OASS-ligand interactions. Substrate binding is more sensitive to salt concentrations than product binding. Our studies reveal that binding of substrate and product exhibit completely different thermodynamic signatures. Product binding is driven by both favorable enthalpic and entropic contributions whereas substrate binding is predominantly driven by entropic contribution.

\section{Results}

\section{Characterization of ligand binding of OASS by fluorescence spectroscopy}

The absorbance spectra of OASS from all three species (H. influenza, S. typhimurium, M.tuberculosis) showed two distinct absorption maxima, one around $280 \mathrm{~nm}$ and a second maximum at $412 \mathrm{~nm}$ indicative of the presence of internal aldimine [17]. Excitation of OASS at $292 \mathrm{~nm}$ yields a fluorescence emission 325-350 nm due to Trp emission and excitation at $412 \mathrm{~nm}$ gives another emission spectrum in the range 475-540 nm due to PLP fluorescence. Fluorescence properties of OASS were investigated previously and it was observed that excitation at $298 \mathrm{~nm}$ generates a large increase in fluorescence between 340-345 $\mathrm{nm}$ [24,25]. Similar to earlier 
observations, excitation at $292 \mathrm{~nm}$ yields an emission spectrum centered at $345 \mathrm{~nm}$ and fluorescence at 345 $\mathrm{nm}$ is quenched upon ligand binding (Figure 1A). We have examined the binding of O-acetyl serine (OAS), Lcysteine, L-methionine, L-serine, and L-isoleucine to OASS by monitoring the changes in fluorescence properties of the protein. Binding of cysteine or methionine to OASS changes both tryptophan and PLP fluorescence spectra (Figure $1 \mathrm{~A} \& 1 \mathrm{~B}$ ). Ligand binding quenches tryptophan fluorescence observed at $345 \mathrm{~nm}$ (Figure 1A), but increases the PLP fluorescence at 507 $\mathrm{nm}$ as observed earlier [26]. It was observed that
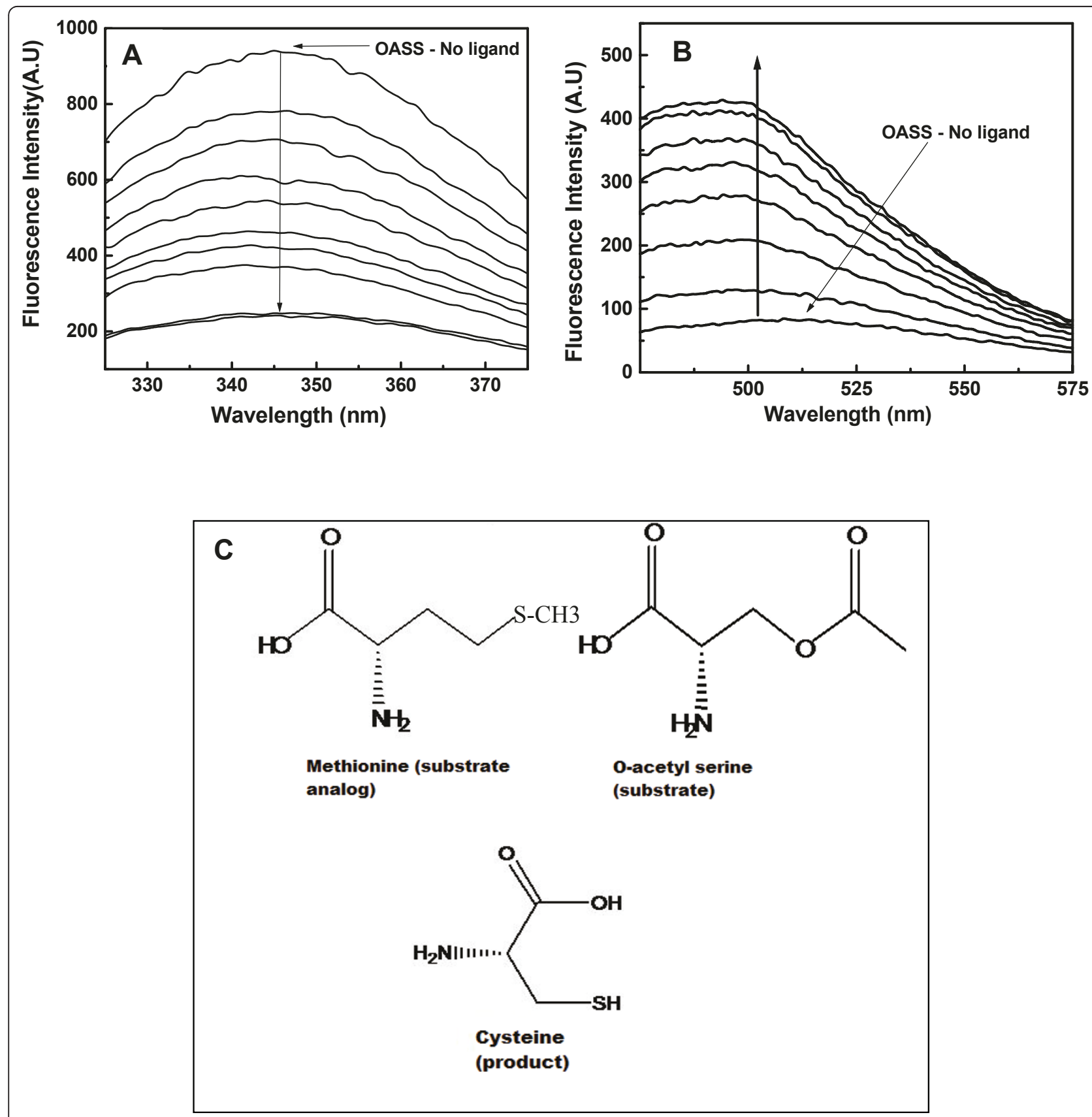

Figure 1 Spectroscopic characterization of OASS and ligand binding. (A) Fluorescence emission of OASS upon excitation at $295 \mathrm{~nm}$ and quenching of fluorescence due to ligand binding (down arrow indicates decrease in fluorescence upon addition of ligands). (B) Fluorescence emission of OASS upon excitation at $412 \mathrm{~nm}$ (PLP) and change in fluorescence intensity due to methionine binding (upward arrow indicates increase in PLP fluorescence upon addition of ligands). Fluorescence increase at $507 \mathrm{~nm}$ was used for determining the extent of binding. (C) Cartoon representation of substrate (OAS), substrate analogue (methionine), and product (cysteine) used in this study. 
excitation of OASS at $290 \mathrm{~nm}$ leads to fluorescence at $345 \mathrm{~nm}$ as well as at $500 \mathrm{~nm}$ due to energy transfer from tryptophan to PLP. Therefore, we monitored the increase in the PLP fluorescence emission at $507 \mathrm{~nm}$ upon excitation at $412 \mathrm{~nm}$ for constructing binding isotherms and determining the equilibrium binding parameters. Addition of OAS quenches the PLP fluorescence initially, but fluorescence signal increases as a function of time indicating the existence of a slow kinetic process after initial quenching (Figure 2A). Detailed studies are necessary to understand the molecular origin of this slow kinetic process.

Binding of methionine and cysteine to OASS results in the formation of external aldimines [27]. Pre-steady state studies observed the slow disappearance of $\alpha$-aminoacrylate indicating that $\alpha$-aminoacrylate may be hydrolyzed in the absence of sulfide [27]. Therefore, it is possible that slow increase of PLP fluorescence may represent hydrolysis of $\alpha$-aminoacrylate or yet other uncharacterized processes. Methionine has been used as substrate analogue in earlier studies to understand the reaction mechanism [15]. Methionine forms stable external aldimine upon binding to OASS and yields a stable signal change which could be used for studying the interaction quantitatively.

In each titration, the relative PLP fluorescence increases with the addition of more ligands reaching a plateau upon saturation of binding sites on OASS (Figure 1B). Since OASS is a homo-dimer, we fitted all the binding isotherms in this study to a two-site binding model (eq1). Fitting yields similar values for $K_{1 \text {,obs }}$ and $\mathrm{K}_{2 \text {,obs }}$ for each binding site. Binding of cysteine to StOASS yields $\mathrm{K}_{1, \mathrm{obs}}$ and $\mathrm{K}_{2 \text {,obs }}=6.0 \pm 0.1 \times 10^{2} \mathrm{M}^{-1}$ $\left(\mathrm{K}_{\mathrm{d}} \sim 1.7 \mathrm{mM}\right)$. Binding of methionine to StOASS yields $\mathrm{K}_{1, \mathrm{obs}}$ and $\mathrm{K}_{2, \mathrm{obs}}=2.9 \pm 0.1 \times 10^{2} \mathrm{M}^{-1}\left(\mathrm{~K}_{\mathrm{d}} \sim 3.4 \mathrm{mM}\right)$ and binding parameters for $\mathrm{HiOASS}$ and $\mathrm{MtOASS}$ are shown in table 1. Equilibrium binding constants obtained for methionine binding from our studies are similar to equilibrium constants estimated for OAS binding using single-wavelength stopped flow studies $\left(\mathrm{K}_{\text {ext }}=0.18 \mathrm{mM}^{-1} \sim 1.8 \times 10^{2} \mathrm{M}^{-1} ; \mathrm{K}_{\mathrm{d}} \sim 5.6 \mathrm{mM}\right)$ [25]. Therefore, we studied the binding of methionine and cysteine for understanding the ligand recognition mechanisms of OASS (Figure 1C). First, we tested the secondary structural properties of OASS in the presence of these ligands. We compared secondary structural contents of unliganded enzyme and ligand bound forms using circular dichroism method. Results of CD spectra for both cysteine and methionine bound OASS are similar to that of unliganded OASS spectrum (Additional file 1, Figure S1). Crystal structure of StOASS-methionine complex showed that active site of OASS is in the closed form as compared to the structure of unliganded enzyme $[14,15]$. Secondary structural contents of both closed and open forms are almost similar as observed from crystal structures of unliganded and complex forms. We have also performed titrations of L-serine and L-isoleucine under similar experimental conditions. In contrast to earlier observation, addition of L-serine does not change OASS fluorescence under our solution conditions $[17,27]$. We found that increasing the $\mathrm{pH}$ of buffer to 9.5 leads to changes in the OASS fluorescence upon addition of L-serine (Additional file 1, Figure S2). We monitored the fluorescence signal change for determining the binding constant. Analysis of L-serine binding isotherm yields a $K_{d}$ value of $\sim 3.7 \mathrm{mM}$ which is less than the reported $K_{d}$ value of $4.2 \mathrm{mM}$ from earlier study [17]. This difference may arise from differences in solution conditions (Additional file 1, Figure S2 and S3). Next, we studied the binding of isoleucine to OASS. OASS has been shown to interact with the C-terminal tail of serine acetyltransferase (SAT) [3]. The last residue at the C-termini of SAT is the highly conserved isoleucine and replacing this isoleucine with alanine decreases the affinity of SAT to OASS significantly [3]. We tested the possibility that OASS may specifically recognize isoleucine when presented as amino acid. Although isoleucine is the most important residue in determining the affinity between OASS and SAT, it does not bind OASS when presented as amino acid.

\section{Determination of OASS-ligand interaction energetics by ITC}

To the best of our knowledge, ITC has not been used to study ligand binding properties of OASS for quantifying the interaction parameters for OASS-ligand complex formation. The energetics of OASS binding to OAS, cysteine, methionine, serine, and isoleucine were examined. We performed isothermal titration calorimetry experiments at $25^{\circ} \mathrm{C}$ to probe the thermodynamics of OAS binding to OASS. Two titrations performed by injecting OAS to solution containing StOASS and $\mathrm{HiOASS}$ are shown in Figure $3 \mathrm{~A}$ and $3 \mathrm{~B}$. Heat signals were integrated, and the binding isotherm was analyzed using two independent site model. Two independent titrations show that binding of OAS is an exothermic reaction and binding isotherms can be fitted to the same model. The analysis of OAS binding to StOASS yielded an enthalpy value of $0.43 \pm 0.2 \mathrm{~kJ} / \mathrm{M}$ and an apparent binding constant of $\sim 1.8 \pm 0.6 \times 10^{3} \mathrm{M}^{-1}(\Delta \mathrm{G}$ $\left.=-17.6 \mathrm{~kJ} / \mathrm{M} ; \mathrm{K}_{\mathrm{d}}=0.56 \mathrm{mM}\right)$. Similarly, analysis of interactions of other two OASS with OAS yielded enthalpy values of $0.45 \pm 0.3 \mathrm{~kJ} / \mathrm{M}$ (HiOASS) and $0.53 \pm$ $0.3 \mathrm{~kJ} / \mathrm{M}$ (MtOASS). The binding constants obtained for both $\mathrm{HiOASS}$ and $\mathrm{MtOASS}$ were similar $(\sim 1.3 \pm 0.4 \times$ $10^{3} \mathrm{M}^{-1} ; \Delta \mathrm{G}=-16.9 \mathrm{~kJ} / \mathrm{M} ; \mathrm{K}_{\mathrm{d}} \sim 0.77 \mathrm{mM}$ for $\left.\mathrm{HiOASS}\right)$. In all three cases, the enthalpy contributes less than $3 \%$ to the total binding free energy. This is surprising 

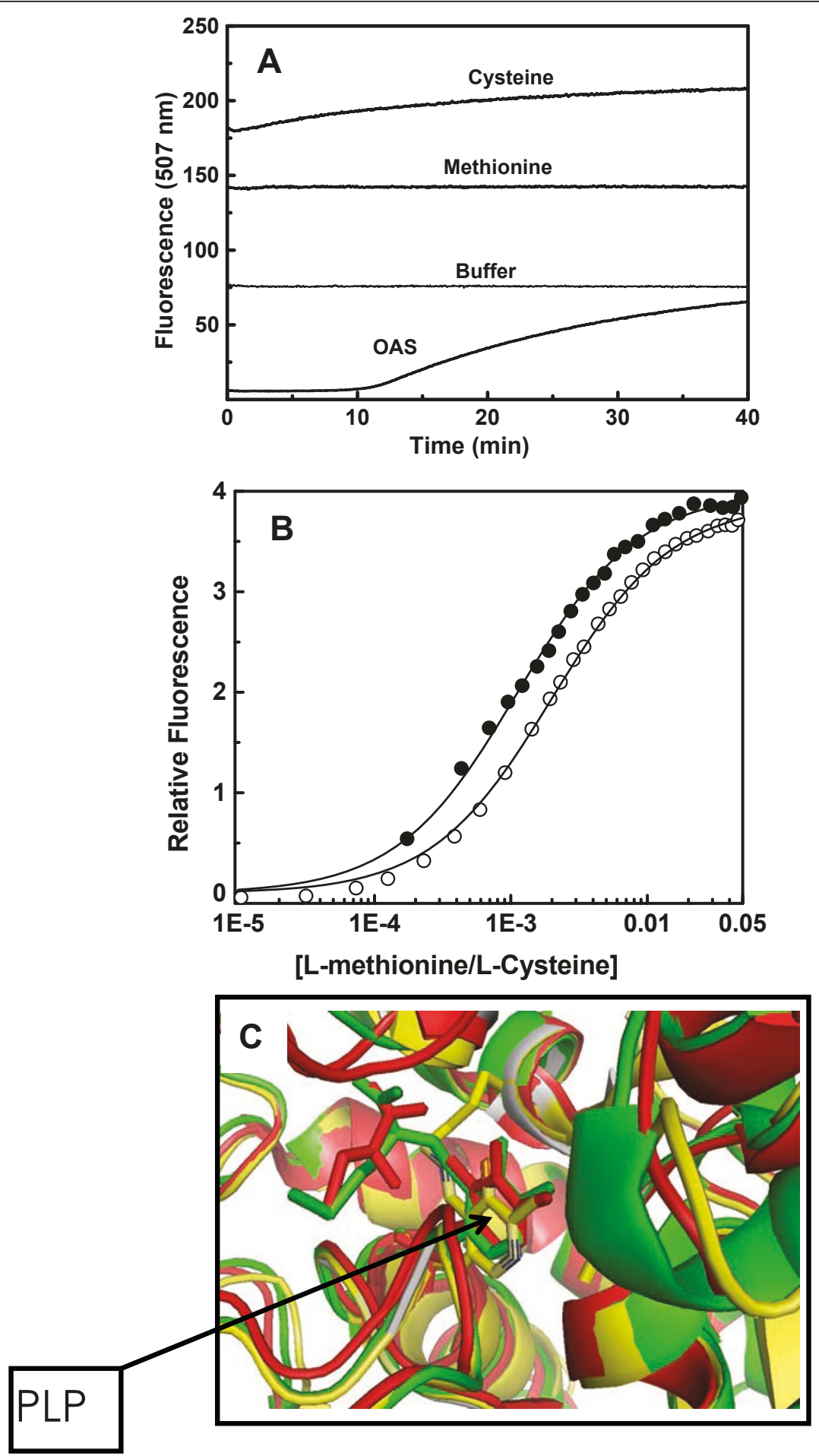

Figure 2 Monitoring ligand binding by fluorescence spectroscopy. (A) Time dependent PLP fluorescence of OASS (1 $\mu M)$ after addition of buffer, OAS $(4 \mu \mathrm{M})$, methionine $(1.4 \mathrm{mM})$, and cysteine $(1.3 \mathrm{mM})$ as indicated in the figure. (B) Fluorescence quenching titrations of the StOASS with cysteine (closed circle) and methionine (open circle); the data from both sets were fit to two identical site model (eq 1) independently. The solid line represents the best fit to data that yields $\mathrm{K}_{\text {cysteine }}=6.0 \times 10^{2} \mathrm{M}^{-1} ; \mathrm{K}_{\text {met }}=2.9 \times 10^{2} \mathrm{M}^{-1} ;$ (C) OASS-cysteine (red) and OASS-met (Green) complexes superimposed to show the interaction of ligand in the active site. Methionine is covalently attached to the active site PLP through Schiff base. 
Table 1 Thermodynamic parameters associated with binding of cysteine and methionine to OASS

\begin{tabular}{|c|c|c|c|c|c|c|}
\hline & & Cysteine & & Methionine & & \\
\hline Temperature & $\Delta \mathrm{G}(\mathrm{kJ} / \mathrm{mol})$ & $\Delta \mathrm{H}(\mathrm{kJ} / \mathrm{mol})$ & TdS (kJ/mol) & $\Delta \mathrm{G}(\mathrm{kJ} / \mathrm{mol})$ & $\Delta \mathrm{H}(\mathrm{kJ} / \mathrm{mol})$ & $\mathrm{TdS}(\mathrm{kJ} / \mathrm{mol})$ \\
\hline \multicolumn{7}{|l|}{ StOASS binding } \\
\hline $10^{\circ} \mathrm{C}$ & $-9.6 \pm 0.4$ & $-2.7 \pm 0.1$ & 6.9 & $-8.6 \pm 0.8$ & $-1.3 \pm 0.1$ & 7.3 \\
\hline $15^{\circ} \mathrm{C}$ & $-9.6 \pm 0.3$ & $-2.8 \pm 0.1$ & 6.8 & $-8.6 \pm 0.4$ & $-1.4 \pm 0.1$ & 7.2 \\
\hline $20^{\circ} \mathrm{C}$ & $-9.8 \pm 0.1$ & $-6.4 \pm 0.1$ & 3.4 & $-9.7 \pm 0.5$ & $-1.5 \pm 0.1$ & 8.2 \\
\hline $25^{\circ} \mathrm{C}$ & $-10.6 \pm 0.1$ & $-5.9 \pm 0.1$ & 4.7 & $-9.5 \pm 0.5$ & $-0.9 \pm 0.1$ & 8.6 \\
\hline $30^{\circ} \mathrm{C}$ & $-10.2 \pm 0.2$ & $-4.4 \pm 0.2$ & 5.8 & $-10.4 \pm 0.8$ & $-1.0 \pm 0.1$ & 9.4 \\
\hline $35^{\circ} \mathrm{C}$ & $-11.0 \pm 0.2$ & $-5.4 \pm 0.3$ & 5.6 & $-11.3 \pm 1.3$ & $-1.2 \pm 0.1$ & 10.1 \\
\hline \multicolumn{7}{|l|}{ HiOASSbinding } \\
\hline $10^{\circ} \mathrm{C}$ & $-8.3 \pm 0.4$ & $-4.6 \pm 0.1$ & 3.7 & $-7.2 \pm 0.9$ & $-0.2 \pm 0.1$ & 7.1 \\
\hline $15^{\circ} \mathrm{C}$ & $-8.7 \pm 0.2$ & $-4.6 \pm 0.2$ & 4.1 & $-7.2 \pm 0.9$ & $-0.2 \pm 0.1$ & 7.5 \\
\hline $20^{\circ} \mathrm{C}$ & $-9.5 \pm 0.5$ & $-4.4 \pm 0.2$ & 5.1 & $-8.2 \pm 1.0$ & $-0.2 \pm 0.1$ & 8.0 \\
\hline $25^{\circ} \mathrm{C}$ & $-9.5 \pm 0.5$ & $-5.4 \pm 0.3$ & 4.1 & $-7.6 \pm 1.1$ & $-0.8 \pm 0.1$ & 6.9 \\
\hline $30^{\circ} \mathrm{C}$ & $-11.0 \pm 0.3$ & $-4.5 \pm 0.4$ & 6.5 & $-8.4 \pm 1.0$ & $-0.7 \pm 0.1$ & 7.7 \\
\hline
\end{tabular}

Titrations were performed in $20 \mathrm{mM}$ Tris buffer $(\mathrm{pH} 8.0), 50 \mathrm{mM} \mathrm{NaCl}$. Data were fit to a two independent sites binding model that yielded similar values of $\Delta \mathrm{G}$, $\Delta \mathrm{H}$, and $-\mathrm{T} \Delta \mathrm{S}$ for each site.

because OAS is a small metabolite and has higher degrees of freedom in solution as compared to the bound form. Therefore, the entropy change upon complex formation is expected to be unfavorable for OASenzyme interaction. On the contrary, OAS interaction which includes its initial reaction and subsequent binding is predominantly driven by favorable entropy component in both cases.

To compare the entropy driven binding of OAS with other ligands, we performed titrations using methionine (substrate analogue), and cysteine, the product of OASS. Titrations performed at $25^{\circ} \mathrm{C}$ indicate that binding of both cysteine and methionine to StOASS is exothermic (Figure 4A and 4B). Analysis of binding isotherms indicated that isotherms can be fitted to a two-sites binding model and fitting yielded similar values for each binding site (table 2). Interestingly, the binding reaction was observed to be entropically driven for methionine binding $\left(\mathrm{T} \Delta \mathrm{S}_{\mathrm{St}-\mathrm{Met}}=8.2 \mathrm{~kJ} / \mathrm{M}\right)$ with little contribution from enthalpy $\left(\Delta \mathrm{H}_{\text {St-Met }}=-1.5 \pm 0.1 \mathrm{~kJ} / \mathrm{M}\right)$ at $20^{\circ} \mathrm{C}$ (table 1$)$. In contrast, cysteine binding is accompanied by both favorable enthalpic and entropic contributions $\left(\Delta \mathrm{H}_{\mathrm{St}-\mathrm{Cys}}\right.$ $\left.=-6.4 \pm 0.1 \mathrm{~kJ} / \mathrm{M} ; \mathrm{T} \Delta \mathrm{S}_{\mathrm{St}-\mathrm{Cys}}=3.4 \mathrm{~kJ} / \mathrm{M}\right)$ at $20^{\circ} \mathrm{C}$. In summary, our results indicate that both OAS and methionine binding is driven by favorable entropy whereas the cysteine binding is driven by both enthalpy and entropy.

\section{Temperature dependence of cysteine and methionine binding to StOASS}

To study the differential recognition of substrate and product by OASS, binding reaction was studied as a function of temperature $\left(10-37^{\circ} \mathrm{C}\right)$. Titration of OAS at different temperatures and comparison and analysis of binding isotherms was difficult. OAS is an activated molecule, and its stability is temperature dependent. Although titration of OAS at $15^{\circ} \mathrm{C}$ provides binding isotherm, titrations at $30^{\circ} \mathrm{C}$ and $35^{\circ} \mathrm{C}$ show low signal to noise and are difficult to analyze (data not shown). Repeated experiments did not yield analyzable quality isotherms. Therefore, we examined the binding of methionine and cysteine as a function of temperature.

The binding isotherms obtained at different temperatures were all exothermic and binding was accompanied with significant contributions from enthalpy and entropy. All binding isotherms were fit using a twoindependent binding sites model. Binding enthalpy increases as temperature is raised and dependence of enthalpy on temperature yields a net heat capacity change $\left(\Delta \mathrm{C}_{\mathrm{p}}=-130 \mathrm{JK}^{-1} \mathrm{M}^{-1}\right)$ for StOASS-cysteine complex formation. To investigate the predominantly entropy driven binding of methionine to OASS, we studied the StOASS-methionine interaction as a function of temperature to examine whether entropy remains as the major driving force at different temperatures $\left(10-37^{\circ} \mathrm{C}\right)$. Although the methionine binding examined at all temperatures exhibited exothermic reaction similar to cysteine binding, binding at each temperature is accompanied with relatively large favorable entropic changes (table 1). Methionine binding reaction to StOASS shows that net enthalpic contribution is only $\sim 10 \%$ of the total binding free energy $\left(\Delta \mathrm{H}_{\mathrm{St}-\mathrm{Met}}=-1.0 \pm 0.2 \mathrm{~kJ} / \mathrm{M}\right.$; $\left.\Delta \mathrm{G}_{\mathrm{St}-\mathrm{Met}}=-10.4 \mathrm{~kJ} / \mathrm{M}\right)$. In contrast, both enthalpic and entropic factors significantly contribute to cysteine binding. It is possible that linked process such as protonation or deprotonation can contribute to these 

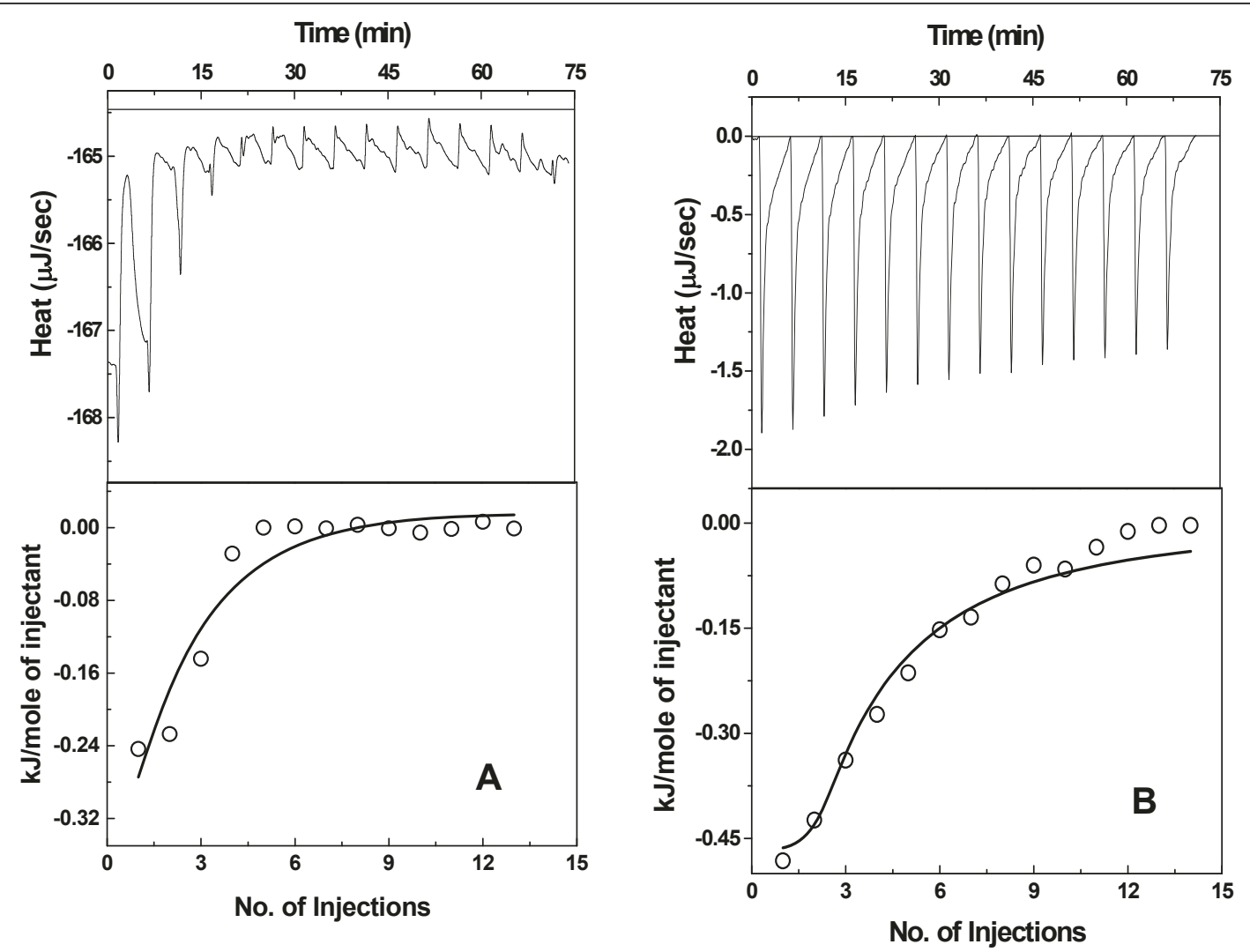

Figure 3 ITC analysis of the interaction between substrate, OAS (O-acetylserine) and OASS. Data is plotted as heat signal ( $\mu$ J/sec) versus time ( $\mathrm{min}$ ) in the upper panel in each fig. Lower panel-integrated heat responses per injection from panel A plotted as normalized heat per mole of injectant. The solid line represents the best fit of the data to a two independent site binding model (eqn 2). (A) Titration of StOASS (4.5 $\mu \mathrm{M})$ with OAS $(5 \mathrm{mM})$ at $25^{\circ} \mathrm{C}$. (B) Titration of HiOASS $(4.5 \mu \mathrm{M})$ with OAS $(5 \mathrm{mM})$ at $25^{\circ} \mathrm{C}$. Both titrations were performed in the same buffer $(20$ $\mathrm{mM}$ Tris, $\mathrm{pH}$ 8.0, $50 \mathrm{mM} \mathrm{NaCl})$.

differences. Dependence of observed enthalpy on ionization enthalpy of the buffer is the test for ligand binding coupled to proton exchange. To detect the potential contribution of protonation changes to the enthalpy of binding, we performed the titration of methionine to StOASS at pH 8.0 in HEPES buffer which has different ionization enthalpy ( $25 \mathrm{mM}$ HEPES, $50 \mathrm{mM} \mathrm{NaCl}$, at $25^{\circ} \mathrm{C}$ ). Binding of methionine in HEPES buffer yields similar enthalpic contribution $\left(\Delta \mathrm{H}_{\mathrm{St}-\mathrm{Met}}=-0.4 \pm 0.1 \mathrm{~kJ} /\right.$ $\mathrm{M})$ suggesting that proton exchange does not contribute to the observed methionine binding enthalpy. Similarly, cysteine-StOASS interaction was also examined at $25^{\circ} \mathrm{C}$ under similar conditions (25 mM HEPES, pH 8.0, 50 $\left.\mathrm{mM} \mathrm{NaCl}, 25^{\circ} \mathrm{C}\right)$. Analysis of cysteine binding isotherm yielded an enthalpic value of $\sim-4.0 \pm 0.2 \mathrm{~kJ} / \mathrm{M}\left(\Delta \mathrm{H}_{\mathrm{St}-\mathrm{Cys}}\right.$ $=-3.9 \mathrm{~kJ} / \mathrm{M})$. Therefore, the higher enthalpic contribution of cysteine binding does not result from linked proton exchange equilibria, but it is the molar enthalpy of cysteine-OASS interaction. Although both cysteine and methionine bind to the same active site and have been shown to form external aldimine, thermodynamic parameters are qualitatively very different. Temperature dependence of binding constants show that van't Hoff plot is linear and cysteine binding shows more temperature dependency than methionine binding (Figure 5A). Temperature dependence of free energy change, enthalpy and entropy are plotted as a function of temperature in Figure 5B \&5C, and dependency of binding constants are shown (Additional file 1, Figure S4). Temperature dependent enthalpy change of cysteine-StOASS interaction is not linear. However, both temperature dependent plots of enthalpy and entropy (Figure 5B $\& 5 \mathrm{C})$ show that methionine and cysteine binding parameters show clear dichotomy.

\section{lonic dependence of ligand binding to OASS}

We have examined the interaction of methionine and cysteine with OASS under different solution conditions. Predominantly entropy driven methionine binding suggests that substrate binding may follow classical hydrophobic interactions with minimal contributions from other forces. On contrary, analyses of structure of 

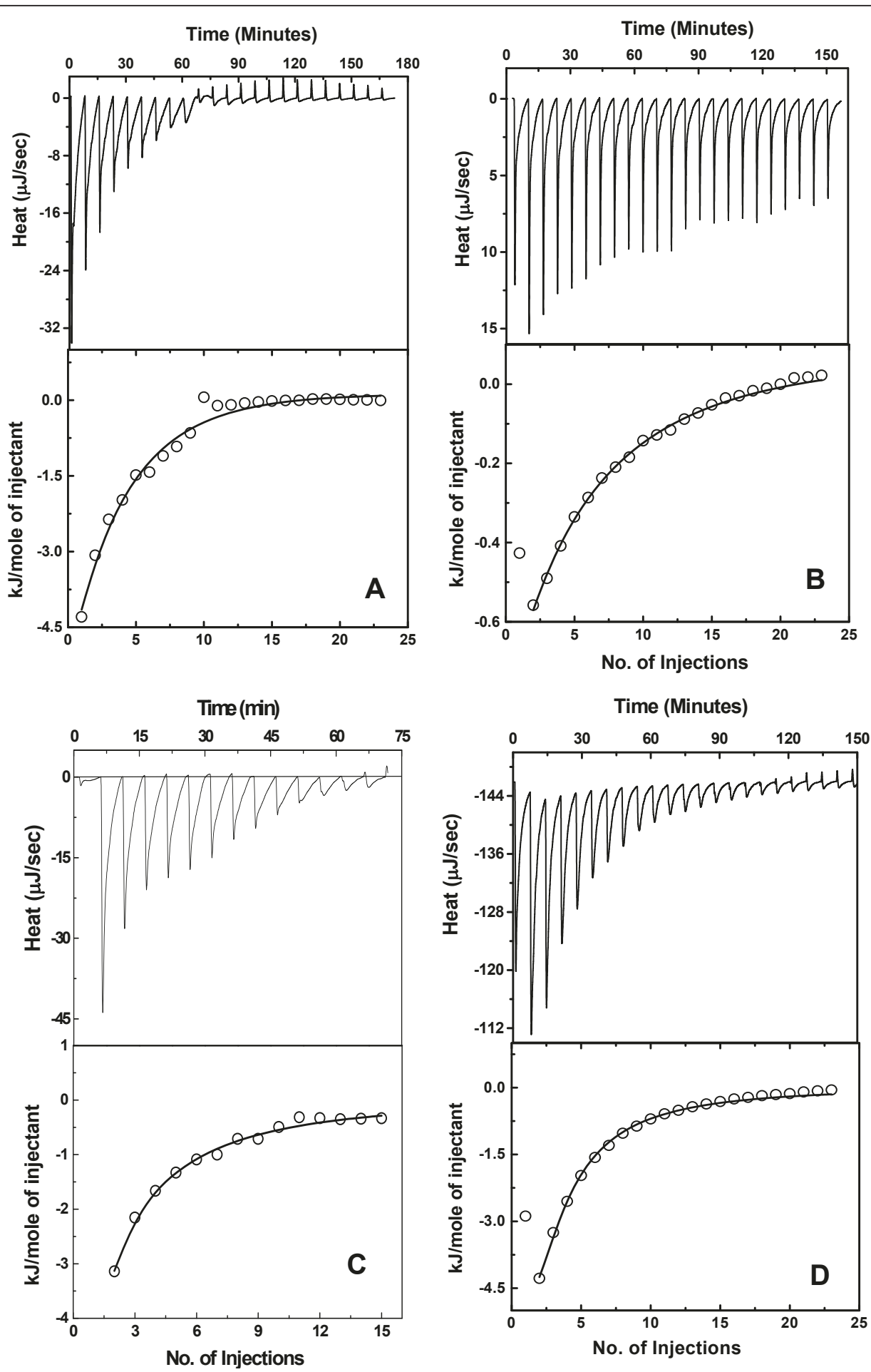

Figure 4 ITC analysis of the interaction between OASS and cysteine. Data is plotted as heat signal ( $\mu \mathrm{J} / \mathrm{sec}$ ) versus time (min) in the upper panel in each fig. Lower panel-Integrated heat responses per injection from panel A plotted as normalized heat per mole of injectant. The solid line represents the best fit of the data to a two independent site binding model (eqn 2). (A) Titration of StOASS (4.5 $\mu \mathrm{M}$ ) with cysteine (350 mM) at $25^{\circ} \mathrm{C}$. (B) Titration of StOASS $(4.5 \mu \mathrm{M})$ with Methionine $(350 \mathrm{mM})$ at $25^{\circ} \mathrm{C}$. (C) Titration of MtOASS $(4.5 \mu \mathrm{M})$ with Cysteine $(350 \mathrm{mM})$ at $25^{\circ} \mathrm{C}$. (D) Titration of HiOASS $(4.5 \mu \mathrm{M})$ with cysteine $(350 \mathrm{mM})$ at $25^{\circ} \mathrm{C}$. 
Table 2 Fluorescence titration analysis of the salt-dependence of interaction between OASS and ligands

\begin{tabular}{|c|c|c|c|c|c|c|c|c|}
\hline \multirow[b]{2}{*}[\mathrm{NaCl}]{$\mathrm{M}$} & \multicolumn{4}{|c|}{ Cysteine } & \multicolumn{4}{|c|}{ Methionine } \\
\hline & $\mathrm{N}$ & Qmax & $\mathrm{K}_{\mathrm{obs}}\left(\mathrm{M}^{-1}\right)$ & $\Delta \mathrm{G}(\mathrm{kJ} / \mathrm{mol})$ & $\mathrm{N}$ & Qmax & $\mathrm{K}_{\mathrm{obs}}\left(\mathrm{M}^{-1}\right)$ & $\Delta \mathrm{G}(\mathrm{kJ} / \mathrm{mol})$ \\
\hline \multicolumn{9}{|c|}{ StOASS binding } \\
\hline 0.02 & 2 & 3.8 & $6.0 \pm 0.1 \times 10^{2}$ & $-15.0 \pm 0.1$ & 2 & 3.8 & $2.9 \pm 0.1 \times 10^{2}$ & $-13.3 \pm 0.1$ \\
\hline 0.05 & 2 & 4.6 & $5.6 \pm 0.1 \times 10^{2}$ & $-14.9 \pm 0.1$ & - & & & \\
\hline 0.1 & 2 & 4.6 & $4.5 \pm 0.1 \times 10^{2}$ & $-14.3 \pm 0.1$ & 2 & 1.0 & $6.5 \pm 0.1 \times 10^{1}$ & $-10.0 \pm 0.1$ \\
\hline 0.2 & 2 & 4.5 & $3.7 \pm 0.1 \times 10^{2}$ & $-13.9 \pm 0.1$ & 2 & 1.4 & $5.5 \pm 0.1 \times 10^{1}$ & $-9.4 \pm 0.1$ \\
\hline 0.5 & 2 & 4.7 & $2.6 \pm 0.1 \times 10^{2}$ & $-13.0 \pm 0.1$ & 2 & 0.5 & $3.5 \pm 0.1 \times 10^{1}$ & $-8.7 \pm 0.1$ \\
\hline \multicolumn{9}{|c|}{ HiOASS binding } \\
\hline 0.02 & 2 & 3.9 & $6.6 \pm 0.1 \times 10^{2}$ & $-15.2 \pm 0.1$ & - & 3.0 & $1.5 \pm 0.1 \times 10^{2}$ & $-11.8 \pm 0.2$ \\
\hline 0.05 & 2 & 5.7 & $5.6 \pm 0.1 \times 10^{2}$ & $-15.5 \pm 0.1$ & - & & & \\
\hline 0.1 & 2 & 5.8 & $4.5 \pm 0.1 \times 10^{1}$ & $-9.0 \pm 0.1$ & 2 & 0.5 & $5.8 \pm 0.1 \times 10^{1}$ & $-9.5 \pm 0.5$ \\
\hline 0.2 & 2 & 5.8 & $3.1 \pm 0.1 \times 10^{1}$ & $-8.0 \pm 0.1$ & 2 & 0.2 & $3.4 \pm 0.1 \times 10^{1}$ & $-8.1 \pm 0.5$ \\
\hline 0.5 & 2 & 3.1 & $3.2 \pm 0.1 \times 10^{1}$ & $-8.0 \pm 0.1$ & 2 & 0.2 & $2.3 \pm 0.1 \times 10^{1}$ & $-7.3 \pm 0.6$ \\
\hline
\end{tabular}

Titrations were performed in $20 \mathrm{mM}$ Tris buffer ( $\mathrm{pH} \mathrm{8.0),} \mathrm{and} \mathrm{indicated} \mathrm{[NaCl].} \mathrm{Data} \mathrm{were} \mathrm{fit} \mathrm{to} \mathrm{a} \mathrm{two} \mathrm{independent} \mathrm{sites} \mathrm{binding} \mathrm{model} \mathrm{that} \mathrm{yielded} \mathrm{similar} \mathrm{values}$ of $K_{\text {obs }}$ for each site.

OASS-methionine complex shows a number of polar interactions between the ligand's charged as well as polar groups and protein side chains suggesting the role of non-hydrophobic forces involved in methionine binding [15]. To understand the dependency of cysteine and methionine binding on electrolyte concentrations, we screened the complex formation in buffers with different ionic strength. Both the methionine and cysteine binding were examined by monitoring the binding processes as a function of $[\mathrm{NaCl}$ (Figure 6A \&6B). Binding of cysteine to StOASS showed that binding constants are not very sensitive to $[\mathrm{NaCl}](\mathrm{d} \log \mathrm{K} / \mathrm{dlog}[\mathrm{NaCl}] \sim-0.21$ $\pm 0.1)$ over the range of $[\mathrm{NaCl}]$ examined $(0.02-0.5 \mathrm{M})$ (table 2). Interestingly, binding of methionine is more $[\mathrm{NaCl}]$ dependent than cysteine binding. Binding constant decreases as $[\mathrm{NaCl}]$ increased with $\operatorname{dlog} \mathrm{K} / \mathrm{dlog}$ $[\mathrm{NaCl}]$ value of $\sim-0.8 \pm 0.1$, which indicates that methionine binding to StOASS is accompanied by the release of one anion or cation (Figure 6C). Comparison of binding constants of cysteine-StOASS and methionine-StOASS complex formation indicates that cysteine has at least 10 fold higher affinity for StOASS than methionine at higher $[\mathrm{NaCl}](0.2-0.5 \mathrm{M})$.

\section{Determination of thermodynamic parameters for MtOASS and HiOASS interaction with ligands}

We have examined the ligand binding properties of OASS from MtOASS and HiOASS to test whether the two different ligand recognition mechanisms observed in this study may be a common feature of bacterial OASS. Both enzymes were cloned and purified as described in materials and methods. Our OAS binding studies have shown that all three enzymes recognize their substrate through entropy dominated mechanism.
Both methionine and cysteine were titrated versus respective OASS under similar conditions. Two independent titrations for each ligand were performed and binding isotherms were fit to two independent binding site model. Representative titrations of MtOASS and $\mathrm{HiOASS}$ binding to cysteine or methionine are shown in Figure 4C \&4D. Binding of both cysteine and methionine to both $M t O A S S$ and HiOASS exhibit features which are very similar to features of StOASS interactions. Binding of both OAS and methionine are predominantly driven by entropy whereas cysteine binding is driven by both enthalpy and entropy. Analyses of binding isotherms yielded the respective thermodynamic parameters and are shown (table 1). The enthalpic contributions for methionine binding to $M t$ OASS and $\mathrm{HiOASS}$ are $5 \%$ and $8 \%$ respectively. Our results presented here clearly show that both substrate and methionine binding to bacterial OASS, in general, may be entropy driven processes.

Since StOASS-ligand interactions showed two distinct binding modes for methionine and cysteine at all temperatures examined, we performed temperature dependent ITC experiments for $\mathrm{HiOASS}$-ligand interactions. Predominantly entropic driven behavior was observed for both StOASS and HiOASS binding to methionine at all temperatures examined. However, specific differences are observed. On average, cysteine binds with higher affinity to StOASS than binding to HiOASS (table 1). In contrast to temperature dependent enthalpy change in StOASS-cysteine interactions, $\mathrm{HiOASS}$-cysteine interaction showed relatively less temperature dependence as compared to StOASS-cysteine interaction (table 1). Hence the heat capacity changes $\left(\Delta \mathrm{C}_{\mathrm{p}}=-130 \mathrm{JK}^{-1} \mathrm{M}^{-1}\right)$ for StOASS-cysteine complex formation is higher than 


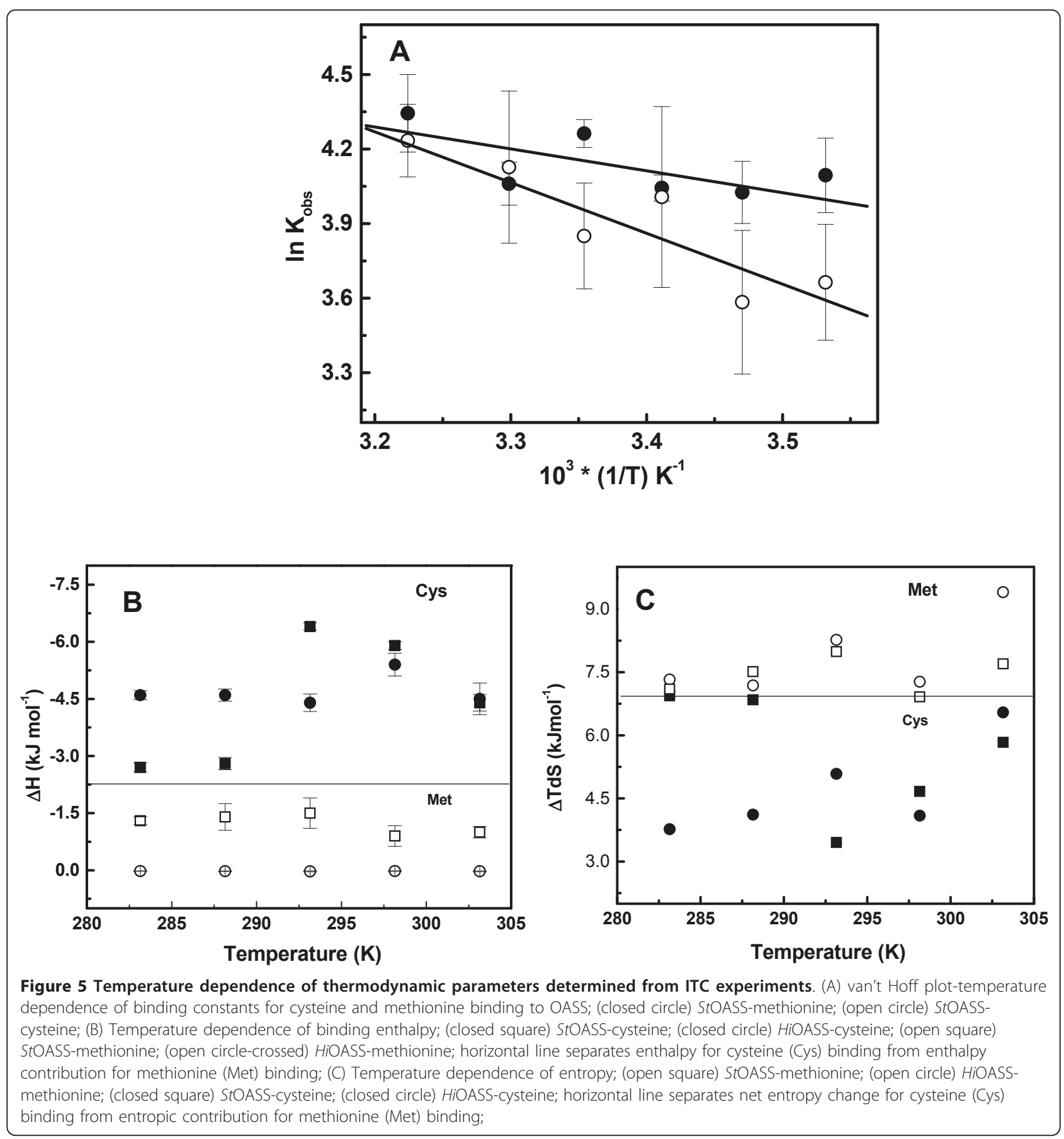

heat capacity changes observed for $\left(\Delta \mathrm{C}_{\mathrm{p}}=-12 \mathrm{JK}^{-1} \mathrm{M}^{-1}\right)$ $\mathrm{HiOASS}$-cysteine complex formation. In contrast, the affinity of cysteine for $\mathrm{HiOASS}$ increases over 10 fold with increasing temperature. Increase in the binding affinity in the case of $\mathrm{HiOASS}$ results from the temperature independent enthalpic contribution as well as increased entropic contribution at higher temperatures (table 1).

\section{Discussion}

Prediction of thermodynamic parameters of proteinligand interactions essentially requires studying the energetics of a large number of protein-ligand interactions in solution in addition to resolving the structures of those protein-ligand complexes. Studies on the ligand recognition features such as multiple binding modes provide information on the dynamic nature of an 

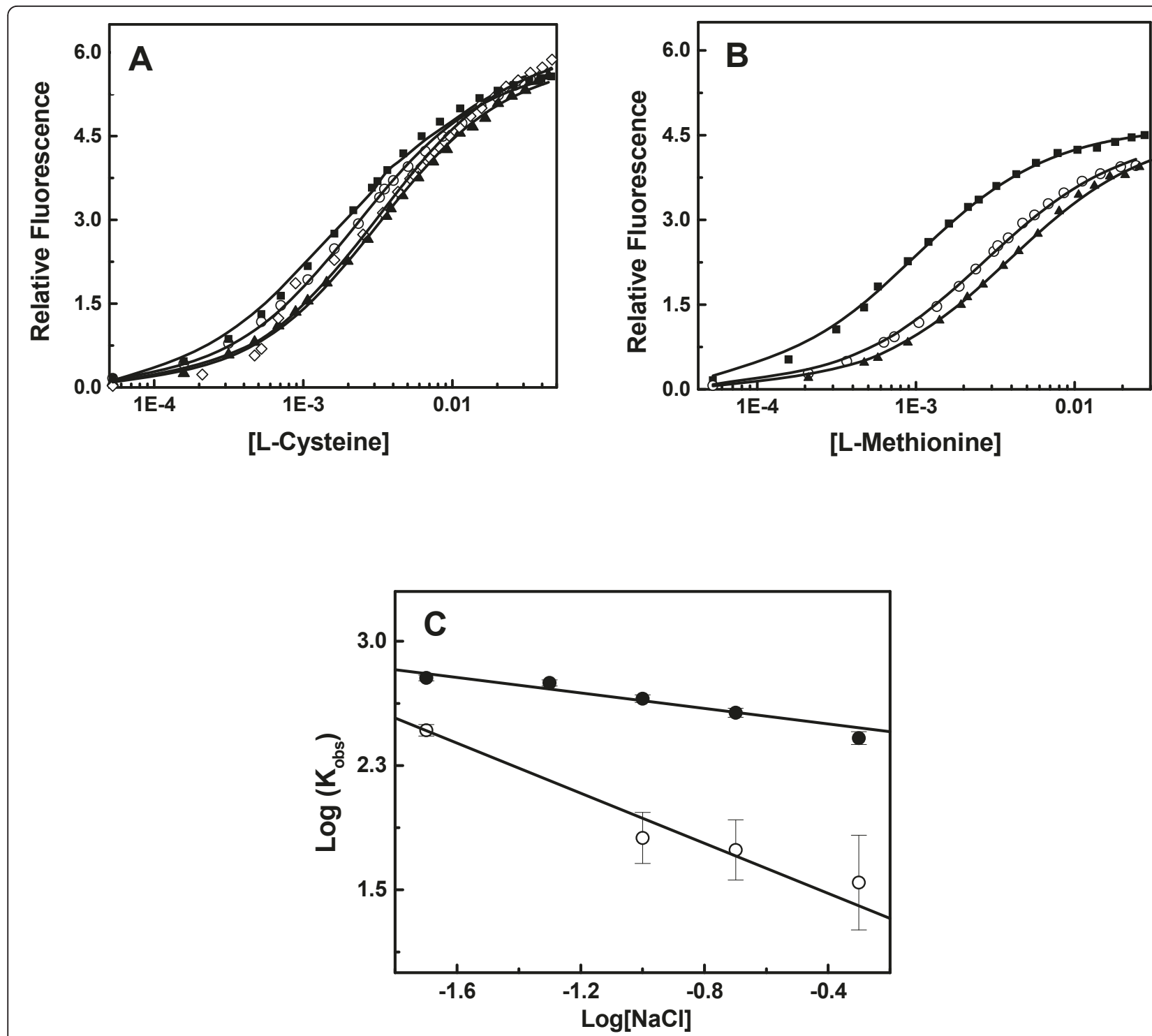

Figure 6 Dependence of ligand-OASS interactions on ionic strength-Fluorescence quenching titrations of ligands in the binding buffer with indicated $[\mathrm{NaCl}]$. Solid line represents the fit derived from two identical site model; (A) Titrations of Cysteine with StOASS at indicated $[\mathrm{NaCl}]$ in the binding buffer; (closed square) 50 mM; (open circle) 100 mM; (open diamond) 200 mM; (closed triangle) 500 mM; (B) Titrations of methionine with StOASS at indicated [NaCl] in the binding buffer; (closed square) 100 mM; (open circle) $200 \mathrm{mM}$; (closed triangle) $500 \mathrm{mM}$; (C) Salt dependence of cysteine (closed circle) and methionine (open circle) binding constants. $\delta \log \left(\mathrm{K}_{\mathrm{obs}}\right) / \delta \log [\mathrm{NaCl}] \sim 0.8$ for methionine binding.

enzyme and essentially required to understand the variable effects of these ligands on enzyme function. Although structural and kinetic studies have enhanced our understanding of catalytic mechanism of OASS, ligand recognition mechanisms are not studied. In the present study, for the first time, we used ITC approaches to determine the thermodynamic parameters associated with the binding of OAS, methionine, and cysteine to OASS from S. typhimurium, M. tuberculosis, and $H$. influenzae. Comparison of detailed binding thermodynamics of three homologous enzymes involved in cysteine biosynthesis with these ligands show that bacterial OASS, in general, may recognize their substrate and product through two different binding mechanisms. In addition, two alternative binding modes for substrate and product binding were observed at all temperatures examined.

Our thermodynamic analyses indicate that binding of OASS to its ligands is accompanied by negative enthalpy change. It is known that formation of hydrogen bonds, hydrophobic, and ionic interactions between protein and ligand could contribute to the observed negative enthalpy change [28]. The favorable entropic contribution could result from desolvation of either ligand/ 
protein binding sites upon interaction or from any conformational changes associated with the binding $[29,30]$. Bound OAS, cysteine, and methionine mediate hydrogen bonds and hydrophobic interactions with the active site residues of OASS. For example, StOASS-methionine structure shows that carboxylate group of methionine interacts with the side chains of THR 68, ASN 69, and GLN 144 [4]. Similarly, both $\alpha$-amino group and carboxylate of cysteine also make several hydrogen bonds with side chain as well as main chain atoms of active site residues $[15,18]$. Based on these structural studies, one may expect that binding of either cysteine or methionine to OASS may proceed through similar mechanism in which both enthalpy and entropy would make significant contributions to the binding free energy. But predominantly entropy driven thermodynamic signatures of substrate and methionine binding suggest that OASS employs entropy dominated mechanisms for recognizing its substrates. Structural studies showed that methionine binding to StOASS induce global conformational changes that lead to closure of the active site [15]. Cysteine binding is also known to induce structural change which is similar to closed form observed for methionine binding. Since the structure of $\mathrm{HiOASS}$-methionine and MtOASS-methionine complex are not available, it is difficult to correlate the structural changes observed in StOASS-methionine bound complex to entropy mediated binding mode observed in our study. Further, lack of structural information on cysteine-OASS complex for StOASS-cysteine and $\mathrm{HiOASS}$-cysteine prevents extrapolation of binding parameters to possible structural changes associated with methionine and cysteine binding.

Predominantly entropy driven binding has been assumed to be driven by classical hydrophobic effect. But cysteine binding to OASS has significant contribution from enthalpy. The hydrophobicity of methionine is slightly higher than that of cysteine [31]. Binding of the OAS also is driven by favorable entropy suggesting that both methionine and OAS are recognized in a similar manner. Methionine and OAS are structurally more similar and contain an additional carbon group which is a methyl in methionine and acetate group in OAS following the electronegative atom (sulfur in methionine and oxygen in the OAS). Therefore, it is conceivable that differential thermodynamic signatures observed for the binding of OAS/methionine and cysteine is due to the structural differences between substrate and product in general. Detailed thermodynamics of ligand binding properties of OASS enzymes from S. typhimurium, $M$. tuberculosis, and $H$. influenzae exhibited qualitatively very different thermodynamic signatures for substrate and product binding. To explain the entropically driven binding, methionine binding was studied as a function of $[\mathrm{NaCl}]$. Our results do not favor the hypothesis that methionine binding is mediated through classical hydrophobic interactions because the binding affinity of methionine shows higher dependency on $[\mathrm{NaCl}]$ than cysteine affinity (table 2).

Entropy driven interactions are shown to result from higher desolvation of the binding interface [30]. Ligand binding induced conformational changes may also be responsible for such behavior if binding induces more disorder to the complex. Binding of methionine is known to change the protein conformation from open to close form, a relative more ordered form [15]. In general, conformational transitions that lead to more disordered form are responsible for favorable entropic contribution. Further, global conformational changes induced by binding would have resulted in non-linear (curved) temperature dependent enthalpy change [32]. The contribution of small conformational alterations, however, cannot be completely ruled out. Therefore, a major fraction of favorable entropy may result from other post-binding related process such as desolvation, protonation, etc. Our results presented here rule out the contribution of proton exchange suggesting that other processes like desolvation may contribute to the observed entropy change. Structures of StOASS complexed with methionine and EhOASS complexed with cysteine show a number of water molecules present in the active site and few of them mediate interactions between ligands and proteins $[15,18]$. Since methionine and OAS have an extra methyl or acetate group, occupation of active site by these bulky ligands may lead to release of more number of non-specifically bound water molecules. Small changes in the structure of ligands (extra methyl group) are proposed to induce considerable changes in the binding [33]. Active site desolvation has been shown to play a role in enzyme catalysis $[30,34]$. The positive effect of substrate induced desolvation on enzyme reactivity has been recognized as an important phenomenon [34]. However, further experimental studies are needed to validate active site desolvation and test its role in OASS activity.

Enzymes involved in de novo cysteine biosynthesis have evolved with multiple regulatory strategies due to the need for tight control over physiological cysteine levels $[1,3,35]$. Therefore physiological activity of OASS is expected to be strictly controlled by temporal flux of metabolites. Equilibrium binding studies on interactions of metabolites with enzymes provide information on binding modes and energetics of interactions which are necessary to understand the regulatory mechanisms of enzymes. OASS has been shown to form non-covalent complexes with both substrate and product $[15,18,23]$. 
Although product inhibition of OASS has not been shown as one of the regulatory inhibition mechanisms, comparable affinity of cysteine for OASS suggest the possibility of product inhibition. At present, cysteine biosynthesis is known to be regulated by two mechanisms $[3,35]$. In the first mechanism, interaction of serine acetyltransferase (SAT) with OASS inhibits cysteine synthesis. The second mechanism operates through feed back inhibition in which cysteine, the product of OASS, inhibits SAT activity and regulates the availability of OAS which is the substrate for OASS. Intracellular cysteine levels are tightly controlled and increase in cysteine levels are known to increase oxidative DNA damage [36]. Considering the key position of OASS in the cysteine biosynthesis pathway, additional regulatory steps other than the two already known mechanisms may exist for fine tuning the cysteine biosynthesis. Alternative binding modes captured in this study may reflect the dynamic nature of this enzyme in recognizing various ligands. Therefore, identifying new molecular features of this enzyme is important to explore yet undiscovered regulatory mechanisms by which OASS may control cysteine flux. Further studies addressing the molecular origin of ligand recognition mechanisms are necessary to underscore the importance of alternative binding modes of OASS.

\section{Conclusions}

For the first time, we have quantified the thermodynamic parameters for OASS binding to its ligands. We found that OASS recognizes its substrate and product through two different mechanisms. Our results presented in this study suggest that substrate binding is predominantly driven by favorable entropic change which may be caused by either active site desolvation or subtle conformational alterations upon binding. We have examined OASS from three different pathogenic bacteria and all three of them show alternative binding modes for substrate and product. Systematic studies on enzyme-ligand interaction studies may reveal more enzymes that may have evolved to recognize substrate and product through different binding modes. Further studies aimed at dissecting molecular origin and structural determinants of substrate and product binding modes are necessary to understand molecular plasticity of enzymes in ligand recognition.

\section{Methods}

\section{Reagents and Buffer}

All chemicals used were reagent grade and buffers were made with double distilled-water. O-acetylserine, Lcysteine, L-serine, L-methionine, and L-isoleucine were obtained from Sigma.

\section{Expression and purification of OASS}

OASS (CysK) genes from S. typhimurium, M. tuberculosis $(H 37 R a)$ and $H$. influenzae were cloned into pET28a $(+)$ vector and vectors containing OASS were transformed in to BL21(DE3) strain for expression. Protein expression was induced using $1 \mathrm{mM}$ IPTG and the induction was carried out at $20^{\circ} \mathrm{C}$ for 16 hours with shaking at $220 \mathrm{rpm}$. Cells were harvested by centrifugation at 4,000 rpm and lysed by sonication. The soluble fraction containing OASS was recovered by centrifugation at 12,000 rpm. The N-terminally His tagged protein was purified using Ni-NTA affinity chromatography followed by gel filtration chromatography on SepharoseS200 column. The purity of OASS was monitored on SDS PAGE followed by Coomassie brilliant blue R-250 staining. The purity of proteins were found to be $>98 \%$. The purified enzymes were active $\left(\mathrm{K}_{\mathrm{m} \text {,OAS }} \sim 0.9-1.4\right.$ $\mathrm{mM} ; k_{\text {cat }} \sim 142, \sim 109 \mathrm{mM} / \mathrm{min} / \mu \mathrm{g}$ for StOASS and HiOASS respectively) and fitting of StOASS of steady state kinetic data is shown (Additional file 2, Figure S1). We have determined the PLP/active-site ratio optically and data is submitted in the supplementary (Additional file 2, Figure S2). Spectroscopic determination of A280/ 412 absorbance ratio for all OASS enzymes used in this study yielded values $\sim 2.6$, as expected for $1: 1$ ratio of PLP bound to one active site [22]. Also we performed activity assays at different enzyme concentrations to check the linearity between enzyme concentration and activity (Additional file 2, Figure S3).

\section{Fluorescence Measurements}

Titrations of ligands with StOASS were examined by monitoring changes in the active site pyridoxal 5'phosphate (PLP) fluorescence of OASS using a Varian spectrofluorometer. The excitation and emission wavelengths for monitoring PLP signal change upon ligand binding were $412 \mathrm{~nm}$ and $507 \mathrm{~nm}$ respectively. All experiments were done at $23.0 \pm 1^{\circ} \mathrm{C}$. Slit widths were set to $5 \mathrm{~nm}$ for all experiments and PMT voltage was adjusted to get maximum signal for a given protein concentration. Initial readings of both the sample, $\mathrm{F}_{\text {samp }, 0}$ and buffer, $\mathrm{F}_{\text {buf }, 0}$ were taken, with $\mathrm{F}_{0}=\mathrm{F}_{\text {samp, } 0^{-}}$ $\mathrm{F}_{\text {buf, } 0}$ defined as the initial fluorescence of the sample. The sample cuvette was then titrated with aliquots of ligands and mixed, equilibrated for 2-5 minutes before measurement. Data points from five such measurements were averaged to obtain $F_{a v e, i}$. The relative fluorescence quenching upon ligand binding is defined as $\mathrm{Q}_{\mathrm{obs}, \mathrm{i}}=$ $\left(\mathrm{F}_{0}-\mathrm{F}_{\mathrm{obs}, \mathrm{i}}\right) / \mathrm{F}_{0}$. All measurements were corrected for dilution, and inner filter effects.

Binding of ligands to OASS was analyzed to obtain the equilibrium binding constant, $\mathrm{K}_{\mathrm{obs}}=[\mathrm{PL}] /[\mathrm{P}]^{*}[\mathrm{~L}]$, using a single or two independent site binding models (eqn 1) 


$$
\mathrm{Q}_{o b s} / \mathrm{Q}_{\max }=\mathrm{n} *\left(K_{o b s} * L\right) /\left(1+K_{o b s} * L\right)
$$

Where n, number of binding sites, $Q_{o b s}$, observed fluorescence quenching and $Q_{\max }$, the maximum fluorescence quenching at saturation.

\section{Calorimetric Measurements}

Isothermal titration calorimetry (ITC) experiments were performed using a Nano-ITC (TA-Instruments). OASS was dialyzed at $25^{\circ} \mathrm{C}$ in $20 \mathrm{mM}$ Tris buffer ( $\mathrm{pH} \mathrm{8.0)}$ and $50 \mathrm{mM} \mathrm{NaCl}$. Buffer solutions were degassed at room temperature prior to use. Briefly, injections of $10 \mu \mathrm{L}$ of either methionine or cysteine dissolved in the dialysis buffer and added using a computer-controlled $250 \mu \mathrm{L}$ microsyringe at an interval of 5-6 minutes into the sample solution containing either StOASS or HiOASS. The heat of dilution for each injection was determined using buffer in the cell. Data obtained from the titration of ligands with OASS were analyzed using either a twosites binding model (eqn 2)

$$
\left.\mathrm{Q}_{\mathrm{i}}{ }^{\text {tot }}=\mathrm{V}_{0} \mathrm{E}_{\text {tot }}\left(\left(\left(K_{1} \mathrm{P}\right) /\left(1+K_{1} \mathrm{P}\right)\right) \Delta \mathrm{H}_{1}\right)+\left(\left(\left(K_{2} \mathrm{P}\right) /\left(1+K_{2} \mathrm{P}\right)\right) \Delta \mathrm{H}_{2}\right)\right)
$$

$\mathrm{Q}_{\mathrm{i}}^{\text {tot }}$ is total heat after the $\mathrm{i}^{\text {th }}$ injection, $\mathrm{V}_{0}$ is the volume of calorimetric cell, $K_{1}$ and $K_{2}$ are the observed equilibrium constants for each site, and $\Delta \mathrm{H}_{1}$ and $\Delta \mathrm{H}_{2}$ are the corresponding enthalpy changes. Estimates of $K_{\text {obs }}$ and $\Delta \mathrm{H}$ were obtained by fitting the experimental data to the model and the best-fit parameters were selected based on the lowest Chi-squared values.

\section{Additional material}

Additional file 1: Spectroscopic characterization of secondary structural contents of OASS and ligand binding. Analyses of secondary structural contents of OASS using CD spectroscopy. L-serine binding studied by fluorescence spectroscopy.

Additional file 2: Spectroscopic and steady-state kinetic characterization of OASS. Catalytic competency of enzyme was analyzed by spectroscopic and kinetic experiments.

\begin{abstract}
Abbreviations
StOASS: Salmonella typhimurium O-acetylserine sulfhydrylase; HiOASS: Haemophilus influenzae O-acetylserine sulfhydrylase; MtOASS: Mycobacterium tuberculosis O-acetylserine sulfhydrylase; EhOASS: Entamoeba histolytica Oacetylserine sulfhydrylase; Tris: tris(hydroxymethyl)aminomethane; ITC: Isothermal Titration Calorimetry; PLP: pyridoxal 5' phosphate.
\end{abstract}

\section{Acknowledgements}

SB and MKE received their fellowships from Council of Scientific and Industrial Research (C.S.I.R). The work was supported by grants to Institute of Microbial Technology from C.S.I.R, Government of India.

\section{Authors' contributions}

SK designed the concepts and experiments of the study. SK, SB cloned the genes. SB expressed, purified and performed all spectroscopic and thermodynamic experiments. MKE performed kinetic experiments. All authors have approved the final manuscript.
Received: 22 January 2011 Accepted: 2 June 2011

Published: 2 June 2011

\section{References}

1. Kredich NM, Becker MA, Tomkins GM: Purification and characterization of cysteine synthetase, a bifunctional protein complex, from Salmonella typhimurium. J Biol Chem 1969, 244:2428-2439.

2. Cook PF, Wedding RT: Initial kinetic characterization of the multienzyme complex, cysteine synthetase. Arch Biochem Biophys 1977, 178:293-302.

3. Kumaran S, Yi H, Krishnan HB, Jez JM: Assembly of the cysteine synthase complex and the regulatory role of protein-protein interactions. $J$ Biol Chem 2009, 284:10268-10275.

4. Rabeh WM, Cook PF: Structure and mechanism of O-acetylserine Sulfhydrylase. J Biol Chem 2004, 279:26803-26806.

5. Bonner ER, Cahoon RE, Knapke SM, Jez JM: Molecular basis of cysteine biosynthesis in plants: structural and functional analysis of $\mathrm{O}_{-}$ acetylserine sulfhydrylase from Arabidopsis thaliana. J Biol Chem 2005, 280:38803-38813.

6. Tai $\mathrm{CH}$, Nalabolu SR, Jacobson TM, Minter DE, Cook PF: Kinetic mechanisms of the $A$ and $B$ isozymes of O-acetylserine sulfhydrylase from Salmonella typhimurium LT-2 using the natural and alternative reactants. Biochemistry 1993, 32:6433-6442.

7. Hell R, Hillebrand $\mathrm{H}$ : Plant concepts for mineral acquisition and allocation. Curr Opin Biotech 2001, 12:161-168.

8. Sekowska A, Kung HF, Danchin A: Sulfur metabolism in Escherichia coli and related bacteria: facts and fiction. J Mol Microbiol Biotechnol 2000, 2:145-177.

9. Cooper AJ: Biochemistry of sulfur-containing amino acids. Annu Rev Biochem 1983, 53:187-222.

10. Wayne LG, Schoskey CD: Non replicating persistence of Mycobacterium tuberculosis. Annu Rev Microbiol 2001, 55:139-163.

11. Wooff E, Michell SL, Gordon SV, Chambers MA, Bardarov S, Jacobs WR Jr, Hewinson RG, Wheeler PR: Functional genomics reveals the sole sulphate transporter of the Mycobacterium tuberculosis complex and its relevance to the acquisition of sulphur in vivo. Mol Microbiol 2002, 43:653-663.

12. Turbull AL, Survette MG: L-Cysteine is required for induced antibiotic resistance in actively swarming $S$. enterica serovar typhimurium. Microbiology 2008, 154:3410-3415.

13. Salsi E, Bayden AS, Spyrakis F, Amadasi A, Campanini B, Bettati S, Dodatko T, Cozzini P, Kellogg GE, Cook PF, Roderick SL, Mozzarelli A: Design of Oacetyl serine sulfhydrylase inhibitors by mimicking nature. J Med Chem 2010, 53:345-356.

14. Burkhard P, Rao GS, Hohenester E, Schnackerz KD, Cook PF, Jansonius JN: Three-dimensional structure of O-acetylserine sulfhydrylase from Salmonella typhimurium. J Mol Biol 1998, 283:121-133.

15. Burkhard P, Tai CH, Ristroph CM, Cook PF, Jansonius JN: Ligand binding induces a large conformational change in O-acetylserine sulfhydrylase from Salmonella typhimurium. J Mol Biol 1999, 291:941-953.

16. Huang $B$, Vetting MW: The active site of O-acetylserine sulfhydrylase is the anchor point for bienzyme complex formation with serine acetyltransferase. J Bacteriol 2005, 187:3201-3205.

17. Schnackerz KD, Tai CH, Simmons JW, Jacobson TM, Rao GS, Cook PF: Identification and spectral characterization of the external aldimine of the O-acetylserine sulfhydrylase reaction. Biochemistry 1995, 34:12152-12160.

18. Chinthalapudi K, Kumar M, Kumar S, Jain S, Alam N, Gourinath S: Crystal structure of native O-acetyl-serine sulfhydrylase from Entamoeba histolytica and its complex with cysteine: structural evidence for cysteine binding and lack of interactions with serine acetyl transferase. Proteins; Struc Funct Bioinform 2008, 72:1222-1232.

19. Schnell R, Oehlmann W, Singh M, Schneider G: Structural insights into catalysis and inhibition of O-acetylserine sulfhydrylase from Mycobacterium tuberculosis: crystal structures of the enzyme aaminoacrylate intermediate and an enzyme-inhibitor complex. J Biol Chem 2007, 282:23473-23481.

20. Cook PF, Wedding RT: A reaction mechanism from steady state kinetic studies for O-acetylserine sulfhydrylase from Salmonella typhimurium LT2. J Biol Chem 1976, 251:2023-2029.

21. Cook PF, Wedding RT: Overall mechanism and rate equation for Oacetylserine sulfhydrylase. J Biol Chem 1977, 252:3459. 
22. Ruben AJ, Kiso Y, Frierie E: Overcoming road blocks in lead optimization: A thermodynamic perpective. Chem Biol Drug Design 2006, 67:2-4

23. Wiseman T, Williston S, Brandts JF, Lin LN: Rapid measurement of binding constants and heats of binding using a new titration calorimeter. Anal Biochem 1989, 179:131-137.

24. Campanini B, Speroni F, Salsi E, Cook PF, Roderick SL, Huang B, Bettati S, Mozzarelli A: Interaction of serine acetyltransferase with O-acetylserine sulfhydrylase active site: Evidence from fluorescence spectroscopy. Protein Sci 2005, 14:2115-2124.

25. Bettati S, Benci S, Campanini B, Raboni S, Chirico G, Beretta S, Schnackerz KD, Hazlett TL, Gratton E, Mozzarelli A: Role of pyridoxal 5'phosphate in the structural stabilization of O-acetylserine sulfhydrylase. J Biol Chem 2000, 275:40244-40251.

26. Rege VD, Kredich NM, Tai CH, Karsten WE, Schnackerz KD, Cook PF: A change in internal aldimine lysine (K42) in O-acetylserine sulfhydrylase to alanine indicates its importance in transimination and as a general base catalyst. Biochemistry 1996, 35:13485-13495.

27. Woehl EU, Tai CH, Dunn MF, Cook PF: Formation of the a-aminoacrylate intermediate limits the overall reaction catalyzed by O-acetyl serine sulfhydrylase. Biochemistry 1996, 35:4776-4783.

28. Hakansson K, Wigley DB: Structure of the complex between a cap analogue and mRNA guanyl transferase demonstrates the structural chemistry of RNA-capping. Proc Natl Acad Sci USA 1998, 95:1505-1510.

29. McElroy C, Manfredo A, Wendt A, Gollnick P, Foster M: TROSY-NMR studies of the $91 \mathrm{kDa}$ protein reveal allosteric control of a gene regulatory protein by ligand-altered flexibility. J Mol Biol 2002, 323:463-473.

30. Shimokhina N, Bronowska A, Homans SW: Contribution of ligand desolvation to binding thermodynamics in a ligand-protein interaction. Angrew Chem Int Ed 2006, 45:6374-6376.

31. Wimley WC, White SH: Experimentally determined hydrophobicity scale for proteins at membrane interfaces. Nat Struct Biol 1996, 10:842-848.

32. Parker AJ: Rates of bimolecular substitution reaction. Adv Phys Org Chem 1967, 5:173-175.

33. Lewi $P J$, de Jonge $M$, Daeyaert $F$, Koymans $L$, Vinkers $M$, Heeres J, Janssen PA, Arnold E, Das K, Clark AD Jr, Hughes SH, Boyer PL, de Béthune MP, Pauwels R, Andries K, Kukla M, Ludovici D, De Corte B, Kavash R, Ho C: On the detection of multiple-binding modes of ligands to proteins from biological, structural, and modeling data. J Comput Aided Mol Des 2003, 17:129-134

34. Cohen SG, Vaidya VM, Schultz RM: Active site of a-chymotrypsin activation by association-desolvation. Proc Natl Acad Sci USA 1970 66:249-256.

35. Droux $M$, Ruffet $M L$, Douce $R$, Job D: Interactions between serine acetyl tranferase and O-acetylserine (thiol) lyase in higher plants-structural and kinetic properties of the free enzyme and bound enzymes. $E J$ Biochem 1998, 255:235-245

36. Park S, Imlay JA: High levels of intracellular cysteine promote oxidative DNA damage by driving the fenton reaction. J Bactrio/ 2003, 185:1942-1950.

doi:10.1186/1471-2091-12-31

Cite this article as: Banerjee et al: Comparative thermodynamic studies on substrate and product binding of O-Acetylserine Sulfhydrylase reveals two different ligand recognition modes ${ }^{\dagger}$. BMC Biochemistry 2011 $12: 31$

\section{Submit your next manuscript to BioMed Central and take full advantage of:}

- Convenient online submission

- Thorough peer review

- No space constraints or color figure charges

- Immediate publication on acceptance

- Inclusion in PubMed, CAS, Scopus and Google Scholar

- Research which is freely available for redistribution 